\title{
New Potential Allelochemicals from Crotalaria Medicaginea Lam.
}

\author{
R.N.YADAVA* and U.K.VISHWAKARMA
}

Natural Products Laboratory, Department of Chemistry, Dr. H. S. Gour University, Sagar (M.P.) 470003, India

rnyadava@rediffmail.com

Received 3 June 2013 / Accepted 10 July 2013

\begin{abstract}
Two new potential allelochemicals have been isolated from methanolic extract of the stems of Crotalaria medicaginea Lam. Along with three known compounds Quercitrin, Acacetin and Isorhamnetin. The structure of two new allelochemicals were characterized as $3,5,7,3^{\prime}, 4^{\prime}$ pentahydroxy-6-methoxyflavone-3- $O-\alpha-L$-rhamnopyranosyl-7- $O-\beta-D$-glucopyranosyl-( $1 \rightarrow 4)-O-\beta$ $D$-xylopyranoside (1) and 3, 5, 7-trihydroxy-8,4'-dimethoxyflavone-5- $O$ - $\beta$-D-galactopyranosyl-7$O-\alpha$ - $L$-rhamnopyranosyl-( $1 \rightarrow 3)-O-\alpha-L$-arabinopyranoside $(2)$ by various colour reactions, spectral analysis and chemical degradations.
\end{abstract}

Keywords: Crotalaria medicaginea Lam., Leguminosae, Allelochemical, Antimicrobial activity

\section{Introduction}

Crotalaria medicaginea Lam. $^{1-3}$ belongs to family leguminosae which is commonly known as "Gulabi" in Hindi. It is found throughout India from the W. Himalaya to Ceylon and Burma. The plant is used in medicine in Punjab. A variety of this species, var. Luxurians, common in western U.P. is considered to be a good camel fodder. The seeds may be used as cattle feed after cooking with common salt. Earlier workers ${ }^{4-8}$ has reported various chemical constituents from this plant. In the present paper we report the isolation and structural elucidation of two new allelochemicals $3,5,7,3^{\prime}, 4^{\prime}$-pentahydroxy-6-methoxyflavone-3- $O$ $\alpha$ - $L$-rhamnopyranosyl-7- $O-\beta$ - $D$-glucopyranosyl-( $(1 \rightarrow 4)-O-\beta$ - $D$-xylopyranoside (1) and 3,5 , 7-trihydroxy-8, 4'-dimethoxyflavone-5- $O-\beta$ - $D$-galactopyranosyl-7- $O-\alpha$ - $L$-rhamnoopyranosyl$(1 \rightarrow 3)-O-\alpha$ - $L$-arabinopyranoside (2) along with three known compounds Quercitrin (3), Acacetin (4) and Isorhamnetin (5) from methanolic extract of stems of this plant.

\section{Experimental}

All of the melting points were determined on a thermoelectrical melting point apparatus and are uncorrected. The IR spectra were recorded in $\mathrm{KBr}$ disc on Perkin Elimer spectrum RX1 $\left(4000-450 \mathrm{~cm}^{-1}\right) .{ }^{1} \mathrm{H}$ NMR and ${ }^{13} \mathrm{C}$ NMR spectra were recorded at $90 \mathrm{MHz}$ using solvent DMSO- $d_{6}$ and TMS as internal standard on Bruker DRX-300 spectrometer. UV spectra were 
recorded in $\mathrm{MeOH}$ (Shimadzu UV 1800 spectrophotometer) and mass spectra on a Jeol SX300 mass spectrometer.

\section{Plant material}

The stems of the plant were collected locally around Sagar region and were taxonomically authenticated by taxonomist, Department of Botany, Dr. H.S. Gour Central University, Sagar (M.P.) India. A voucher specimen has been deposited in the Natural Products Laboratory, Department of Chemistry of this university.

\section{Extraction and isolation}

Air dried and powdered stems $(3 \mathrm{~kg}$ ) of the plant were extracted with methanol in a Soxhlet apparatus for $74 \mathrm{~h}$. The methanolic extract of stems of the plant was further exhaustively partitioned with chloroform, ethyl acetate and acetone. The acetone soluble fraction was further concentrated under reduced pressure to yield brown viscous mass $(2.50 \mathrm{~g})$, which was subjected to TLC examination using nBAW (4:1:5) as solvent and $I_{2}$ vapours as visualizing agent. It gave five spots indicating it to be mixture of five compounds $\mathbf{1}, 2,3,4$ and 5 . These compounds were separated by TLC and purified by column chromatography over silica gel using $\mathrm{CHCl}_{3}: \mathrm{MeOH}$ as eluents and studied separately.

\section{Study of compound 1}

It was crystalysed from acetone to yield 1.45 g. It has m.p. $224-225{ }^{\circ} \mathrm{C}$, m.f. $\mathrm{C}_{33} \mathrm{H}_{40} \mathrm{O}_{21},[\mathrm{M}]^{+}$ 772 (FABMS); found(\%): C 51.18, H 5.26, calcd.(\%) for m.f. $\mathrm{C}_{33} \mathrm{H}_{40} \mathrm{O}_{21}$ :C 51.30, H 5.18; UV $\lambda_{\max } \mathrm{MeOH}(\mathrm{nm}): 260,358,393$; IR: $v_{\max }^{\mathrm{KBr}}\left(\mathrm{cm}^{-1}\right), 3400,2924,2848,1730,1465,1375$, $1269,1025,746,584,537,428 ;{ }^{1} \mathrm{H}$ NMR $\left(90 \mathrm{MHz}, \mathrm{DMSO}-d_{6}\right), \delta(\mathrm{ppm}) ; 6.43(1 \mathrm{H}, \mathrm{s}, \mathrm{H}-8)$, $9.28(1 \mathrm{H}, \mathrm{s}, 3-\mathrm{OH}), 11.40(1 \mathrm{H}, \mathrm{s}, 5-\mathrm{OH}), 10.74(1 \mathrm{H}, \mathrm{s}, 7-\mathrm{OH}), 3.81\left(3 \mathrm{H}, \mathrm{s}, 6-\mathrm{OCH}_{3}\right), 7.70(1 \mathrm{H}$, $\left.\mathrm{d}, J=2.0 \mathrm{~Hz}, \mathrm{H}-2^{\prime}\right), 6.82\left(1 \mathrm{H}, \mathrm{d}, J=8.0 \mathrm{~Hz}, \mathrm{H}-5^{\prime}\right), 7.63\left(1 \mathrm{H}, \mathrm{dd}, J=8.3,2.0 \mathrm{~Hz}, \mathrm{H}-6^{\prime}\right), 8.88$ $\left(1 \mathrm{H}, \mathrm{s}, 3^{\prime}-\mathrm{OH}\right), 8.94\left(1 \mathrm{H}, \mathrm{s}, 4^{\prime}-\mathrm{OH}\right), 5.32\left(1 \mathrm{H}, \mathrm{br}, \mathrm{s}, \mathrm{H}-1^{\prime \prime}\right), 4.20\left(1 \mathrm{H}, \mathrm{m}, \mathrm{H}-2^{\prime \prime}\right), 3.21-3.74(3 \mathrm{H}$, m, H-3", H-4", H-5"), 0.90 (3H, d, $\left.J=5.3 \mathrm{~Hz}, \mathrm{CH}_{3}-6^{\prime \prime}\right), 4.72$ (1H, d, $\left.J=7.1 \mathrm{~Hz}, \mathrm{H}-1^{\prime \prime \prime}\right), 3.21-$ 3.48 (3H, m, H-2"', H-3"', H-4"'), 3.16 (1H, dd, $J=8.9,8.9$ H-5a"'), 3.78 (1H, dd, $J=10.6,5.1$ H-5b"'), 4.62 (1H, d, J=7.6 Hz, H-1"'"), 3.15-3.78 (6H, m, H-2'"', H-3'"', H-4"'"', H-5"'", H$\left.6^{\prime \prime \prime \prime}\right) .{ }^{13} \mathrm{C}$ NMR (90 MHz, DMSO- $\left.d_{6}\right), \delta(\mathrm{ppm}): 157.23(\mathrm{C}-2), 136.4$ (C-3), 179.42 (C-4), 153.54 (C-5), 132.68 (C-6), 157.16 (C-7), 94.78 (C-8), 152.24 (C-9), 105.82 (C-10), 122.64 (C-1'), $116.24\left(\mathrm{C}-2^{\prime}\right), 145.92\left(\mathrm{C}-3^{\prime}\right), 148.85\left(\mathrm{C}-4^{\prime}\right), 116.54\left(\mathrm{C}-5^{\prime}\right), 120.96\left(\mathrm{C}-6^{\prime}\right), 61.26$ (6$\left.\mathrm{OCH}_{3}\right), 102.0\left(\mathrm{C}-1^{\prime \prime}\right), 70.8\left(\mathrm{C}-2^{\prime \prime}\right), 70.6\left(\mathrm{C}-3^{\prime \prime}\right), 70.3\left(\mathrm{C}-4^{\prime \prime}\right), 70.0\left(\mathrm{C}-5^{\prime \prime}\right), 16.2\left(\mathrm{C}-6^{\prime \prime}\right), 106.3(\mathrm{C}-$ $\left.1^{\prime \prime \prime}\right), 75.2$ (C-2'"'), 77.24 (C-3"'), 70.72 (C-4"''), 66.73 (C-5'"'), 102.4 (C-1'"'), 83.2 (C-2'"''), 76.2 (C-3"'"'), 69.3 (C-4"'"'), 77.5 (C-5"'"'), 60.8 (C-6"'"') and [M] 772 (FABMS).

\section{Acid hydrolysis of compound 1}

Compound $1(50 \mathrm{mg})$ was dissolved in ethanol $(15 \mathrm{~mL})$ and refluxed with $20 \mathrm{~mL}$ of $\mathrm{H}_{2} \mathrm{SO}_{4}$ on water bath for 6-8 $\mathrm{h}$. The reaction mixture was concentrated and allowed to cool and residue was extracted with diethyl ether $\left(\mathrm{Et}_{2} \mathrm{O}\right)$. The ether layer was washed with water and evaporated to dryness. The residue was subjected to column chromatography over silica gel column using $\mathrm{CHCl}_{3}$ : $\mathrm{MeOH}$ (3:6) to give compound 1-A, identified as 3, 5, 7, 3', 4'pentahydroxy-6-methoxy flavone by comparison of its spectral data with reported literature values. The aqueous hydrolysate was neutralized with $\mathrm{BaCO}_{3}$ and $\mathrm{BaSO}_{4}$ filtered off. The filtrate was concentrated and subjected to paper chromatography examination using nBAW (4:1:5) solvent and aniline hydrogen phthalate as spraying reagent, showed the presence of $L$-rhamnose $\left(\mathrm{R}_{f} 0.36\right), D$-glucose $\left(\mathrm{R}_{f} 0.19\right)$ and $D$-xylose $\left(\mathrm{R}_{f} 0.27\right)(\mathrm{Co}-\mathrm{PC})$. 


\section{Study of compound 1-A}

It has m.f. $\mathrm{C}_{16} \mathrm{H}_{12} \mathrm{O}_{8}$, m.p. 242-245 ${ }^{\circ} \mathrm{C}, \quad[\mathrm{M}]^{+} 332$ (EIMS); found(\%): C 57.64, H 3.54, calcd (\%) for m.f. $\mathrm{C}_{16} \mathrm{H}_{12} \mathrm{O}_{8}, \mathrm{C}$ 57.83, H 3.61; UV: $\lambda_{\max }(\mathrm{nm})$ : (MeOH) 263, 355, 390; IR: $v_{\max }^{\mathrm{KBr}}\left(\mathrm{cm}^{-1}\right), 3404,2926,2850,1734,1463,1372,1266,1028,742,586,534,426 ;{ }^{1} \mathrm{H}$ NMR (90 MHz, DMSO- $\left.d_{6}\right), \delta(\mathrm{ppm}): 6.45(1 \mathrm{H}, \mathrm{s}, \mathrm{H}-8), 9.32(1 \mathrm{H}, \mathrm{s}, 3-\mathrm{OH}), 12.36(1 \mathrm{H}, \mathrm{s}, 5-\mathrm{OH})$, $10.76(1 \mathrm{H}, \mathrm{s}, 7-\mathrm{OH}), 3.84\left(3 \mathrm{H}, \mathrm{s}, 6-\mathrm{OCH}_{3}\right), 7.72\left(1 \mathrm{H}, \mathrm{d}, J=2.1 \mathrm{~Hz}, \mathrm{H}-2^{\prime}\right), 6.84(1 \mathrm{H}, \mathrm{d}, J=8.1$ $\left.\mathrm{Hz}, \mathrm{H}-5^{\prime}\right), 7.61\left(1 \mathrm{H}, \mathrm{dd}, J=8.4,2.1 \mathrm{~Hz}, \mathrm{H}-6^{\prime}\right), 8.92\left(1 \mathrm{H}, \mathrm{s}, 3^{\prime}-\mathrm{OH}\right), 8.96\left(1 \mathrm{H}, \mathrm{s}, 4^{\prime}-\mathrm{OH}\right) .{ }^{13} \mathrm{C}$ NMR $\left(90 \mathrm{MHz}, \mathrm{DMSO}-d_{6}\right), \delta(\mathrm{ppm}): 147.24$ (C-2), 135.92 (C-3), 176.45 (C-4), 151.93 (C-5), 131.14 (C-6), 157.46 (C-7), 93.72 (C-8), 152.53 (C-9), 103.95 (C-10), 120.78 (C-1'), 115.06 (C-2'), 145.14 (C-3'), 147.74 (C-4'), 115.21 (C-5'), 123.15 (C-6'), $60.12\left(6-\mathrm{OCH}_{3}\right)$.

\section{Permethylation of compound 1}

Compound $1(30 \mathrm{mg})$ was refluxed with MeI $(10 \mathrm{~mL})$ and $\mathrm{Ag}_{2} \mathrm{O}(20 \mathrm{~mL})$ in DMF $(25 \mathrm{mg})$ for two days and then filtered. The filtrate was hydrolyzed with $10 \%$ ethanolic $\mathrm{H}_{2} \mathrm{SO}_{4}$ for 6-7 h, to give methylated aglycone identified as 3, 7-dihydroxy-5, 6, 3', 4'-tetramethoxy flavone and methylated sugars, which were identified as 2, 3, 4 -tri- $O$-methyl- L-rhamnose $\left(\mathrm{R}_{\mathrm{G}} 1.02\right), 2,3$, 4, 6- tetra- $O$-methyl- $D$-glucose $\left(\mathrm{R}_{\mathrm{G}} 1.00\right)$ and 2, 3-di- $O$-methyl- $D$-xylose $\left(\mathrm{R}_{\mathrm{G}} 0.73\right)$.

\section{Enzymatic hydrolysis of compound 1}

Compound $1(25 \mathrm{mg})$ was dissolved in $\mathrm{MeOH}(20 \mathrm{~mL})$ and hydrolysed with equal volume of takadiastase enzyme. The reaction mixture was allowed to stay at room temperature for 2 days and filtered. The proaglycone and hydrolysate were studied separately. The hydrolysate was concentrated and subjected to paper chromatography examination using nBAW (4:1:5) as solvent, which showed the presence of $L$-rhamnose $\left(\mathrm{R}_{f} 0.36\right)(\mathrm{Co}-\mathrm{PC})$. The proaglycone was dissolved in $\mathrm{MeOH}(25 \mathrm{~mL})$ and further hydrolysed with equal volume of almond emulsin enzyme at room temperature as usual procedure yielded aglycone, identified as 3, 5, 7, 3', 4'-pentahydroxy-6-methoxy flavone and sugars were identified as $D$-glucose $\left(\mathrm{R}_{f} 0.82\right)$ and $D$-xylose $\left(\mathrm{R}_{f} 0.27\right)(\mathrm{Co}-\mathrm{PC})$.

\section{Study of compound 2}

It was crystalysed from acetone to yield $950 \mathrm{mg}$. It has m.p. $234-235{ }^{\circ} \mathrm{C}$, m.f. $\mathrm{C}_{34} \mathrm{H}_{42} \mathrm{O}_{20}$, $[\mathrm{M}]^{+} 770$ (FABMS); found(\%): C 52.36, H 5.24, calcd.(\%) for m.f. $\mathrm{C}_{34} \mathrm{H}_{42} \mathrm{O}_{20}: \mathrm{C} 52.99, \mathrm{H}$ 5.45; UV: $\lambda_{\max }(\mathrm{nm}):(\mathrm{MeOH})$ 264, 364; $\left(+\mathrm{AlCl}_{3}\right)$ 268, 425; $\left(+\mathrm{AlCl}_{3} / \mathrm{HCl}\right)$ 270, 426; $(+\mathrm{NaOAc}) 266,370$; IR: $v_{\max }^{\mathrm{KBr}}\left(\mathrm{cm}^{-1}\right), 3484,2906,2873,1656,1623,1591,926,852 ;{ }^{1} \mathrm{H}$ NMR (90 MHz, DMSO- $\left.d_{6}\right), \delta(\mathrm{ppm}) ; 6.15(1 \mathrm{H}, \mathrm{d}, J=2.0 \mathrm{~Hz}, \mathrm{H}-6), 9.44(1 \mathrm{H}, \mathrm{s}, 3-\mathrm{OH})$, $12.48(1 \mathrm{H}, \mathrm{s}, 5-\mathrm{OH}), 10.86(1 \mathrm{H}, \mathrm{s}, 7-\mathrm{OH}), 3.90\left(3 \mathrm{H}, \mathrm{s}, 8-\mathrm{OCH}_{3}\right), 8.15(2 \mathrm{H}, \mathrm{d}, J=8.8 \mathrm{~Hz}, \mathrm{H}-$ $\left.2^{\prime}, \mathrm{H}-6^{\prime}\right), 7.12\left(1 \mathrm{H}, \mathrm{d}, J=8.8 \mathrm{~Hz}, \mathrm{H}-3^{\prime}, \mathrm{H}-5^{\prime}\right), 3.86\left(3 \mathrm{H}, \mathrm{s}, 4^{\prime}-\mathrm{OCH}_{3}\right), 5.23(1 \mathrm{H}, \mathrm{d}, J=7.5, \mathrm{H}-$ $\left.1^{\prime \prime}\right), 3.36-3.72$ (4H, m, H-2", H-3", H-4", H-5" ), 3.80 (1H, dd, $\left.J=11.1,7.1 \mathrm{~Hz}, \mathrm{H}-6_{\mathrm{a}}{ }^{\prime \prime}\right), 4.23$ $\left(1 \mathrm{H}, \mathrm{dd}, J=11.2,4.2 \mathrm{~Hz}, \mathrm{H}-6_{\mathrm{b}}{ }^{\prime \prime}\right), 4.96\left(1 \mathrm{H}, \mathrm{d}, J=6.9 \mathrm{~Hz}, \mathrm{H}-1^{\prime \prime \prime}\right), 3.46-4.42$ (4H, m, H-2"', H-3"', H-4"', H-5"'), 4.54 (1H, d, J 1.21 Hz, H-1"'"), 3.10-3.68 (4H, m, H-2"'", H-3"'"', H$\left.4^{\prime \prime \prime \prime}, \mathrm{H}-5^{\prime \prime \prime \prime}\right), 1.08\left(3 \mathrm{H}, \mathrm{d}, J 6.0 \mathrm{~Hz}, 6{ }^{\prime \prime \prime \prime}-\mathrm{CH}_{3}\right) .{ }^{13} \mathrm{C}$ NMR (90 MHz, DMSO-d $\left.d_{6}\right), \delta(\mathrm{ppm})$ : 144.6 (C-2), 137.2 (C-3), 175.3 (C-4), 155.2 (C-5), 100.6 (C-6), 156.4 (C-7), 132.4 (C-8), 152.8(C-9), 106.7 (C-10), 123.2 (C-1'), $129.3\left(\mathrm{C}-2^{\prime}\right), 114.0\left(\mathrm{C}-3^{\prime}\right), 160.4\left(\mathrm{C}-4^{\prime}\right), 114.7$ $\left(\mathrm{C}-5^{\prime}\right), 129.8\left(\mathrm{C}-6^{\prime}\right), 62.0\left(8-\mathrm{OCH}_{3}\right), 55.8\left(4^{\prime}-\mathrm{OCH}_{3}\right), 104.6\left(\mathrm{C}-1^{\prime \prime}\right), 73.5\left(\mathrm{C}-2^{\prime \prime}\right), 75.6\left(\mathrm{C}-3^{\prime \prime}\right)$, 70.2 (C-4"), 77.6 (C-5"), 62.5 (C-6"), 103.6 (C-1"'), 73.3 (C-2"'), 74.6 (C-3"'), 69.6(C-4"'),

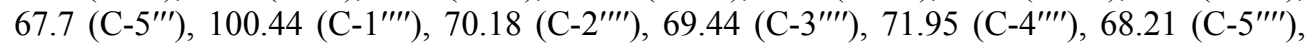
$17.67\left(\mathrm{C}-6^{\prime \prime \prime \prime}\right)$ and $[\mathrm{M}]^{+} 770$ (FABMS). 


\section{Acid hydrolysis of compound 2}

Compound $2(50 \mathrm{mg})$ was dissolved in ethanol $(15 \mathrm{~mL})$ and refluxed with $20 \mathrm{~mL} \mathrm{of} \mathrm{H}_{2} \mathrm{SO}_{4}$ on water bath for 6-8 $\mathrm{h}$. The reaction mixture was concentrated and allowed to cool and residue was extracted with diethyl ether $\left(\mathrm{Et}_{2} \mathrm{O}\right)$. The ether layer was washed with water and evaporated to dryness. The residue was subjected to column chromatography over silica gel column using $\mathrm{CHCl}_{3}$ : $\mathrm{MeOH}$ (3:6) to give compound 2-A, identified as 3, 5, 7-trihydroxy-8, 4'-dimethoxy flavone. The aqueous hydrolysate was neutralized with $\mathrm{BaCO}_{3}$ and $\mathrm{BaSO}_{4}$ filtered off. The filtrate was concentrated and subjected to paper chromatography examination using nBAW (4:1:5) solvent and aniline hydrogen phthalate as spraying reagent, showed the presence of L-rhamnose $\left(\mathrm{R}_{f} 0.36\right), L$-arabinose $\left(\mathrm{R}_{f} 0.22\right)$ and $D$-galactose $\left(\mathrm{R}_{f}\right.$ 0.15) (Co-PC).

\section{Study of compound 2-A}

It has m.f. $\mathrm{C}_{17} \mathrm{H}_{14} \mathrm{O}_{7}$, m.p. $256-257{ }^{\circ} \mathrm{C}, \quad[\mathrm{M}]^{+} 330$ (EIMS); found(\%): C 60.28, H 4.10, calcd (\%) for m.f. $\mathrm{C}_{17} \mathrm{H}_{14} \mathrm{O}_{7}, \mathrm{C}$ 61.82, H 4.24; UV: $\lambda_{\max }(\mathrm{nm})$ : $(\mathrm{MeOH}) 262,366 ;\left(+\mathrm{AlCl}_{3}\right)$ 266, 427; $\left(+\mathrm{AlCl}_{3} / \mathrm{HCl}\right)$ 272, 424; (+NaOAc) 268, 372; IR: $v_{\max }^{\mathrm{KBr}}\left(\mathrm{cm}^{-1}\right), 3486,2904$, $2876,1654,1626,1594,924,854 ;{ }^{1} \mathrm{H}$ NMR $\left(90 \mathrm{MHz}, \mathrm{DMSO}-d_{6}\right), \delta(\mathrm{ppm}): 6.18(1 \mathrm{H}, \mathrm{d}, J$ $=2.1 \mathrm{~Hz}, \mathrm{H}-6), 9.46(1 \mathrm{H}, \mathrm{s}, 3-\mathrm{OH}), 12.45(1 \mathrm{H}, \mathrm{s}, 5-\mathrm{OH}), 10.86(1 \mathrm{H}, \mathrm{s}, 7-\mathrm{OH}), 3.88(3 \mathrm{H}, \mathrm{s}$, $\left.8-\mathrm{OCH}_{3}\right), 8.13\left(2 \mathrm{H}, \mathrm{d}, J=8.9 \mathrm{~Hz}, \mathrm{H}-2^{\prime}, \mathrm{H}-6^{\prime}\right), 7.10\left(2 \mathrm{H}, \mathrm{d}, J=8.9 \mathrm{~Hz}, \mathrm{H}-3^{\prime}, \mathrm{H}-5^{\prime}\right), 3.84$ $\left(3 \mathrm{H}, \mathrm{s}, 4^{\prime}-\mathrm{OCH}_{3}\right) \cdot{ }^{13} \mathrm{C}$ NMR $\left(90 \mathrm{MHz}, \mathrm{DMSO}-d_{6}\right), \delta(\mathrm{ppm}): 146.4(\mathrm{C}-2), 136.3(\mathrm{C}-3)$, 176.2 (C-4), 156.9 (C-5), 99.4 (C-6), 157.6 (C-7), 128.4 (C-8), 150.3(C-9), 104.4 (C-10), $\left.123.5\left(\mathrm{C}-1^{\prime}\right), 129.6\left(\mathrm{C}^{2}\right)^{\prime}\right), 114.3\left(\mathrm{C}-3^{\prime}\right), 160.8\left(\mathrm{C}-4^{\prime}\right), 114.3\left(\mathrm{C}-5^{\prime}\right), 129.6\left(\mathrm{C}-6^{\prime}\right), 61.8$ (8$\left.\mathrm{OCH}_{3}\right), 55.6\left(4^{\prime}-\mathrm{OCH}_{3}\right)$.

\section{Permethylation of compound 2}

Compound 2 (20 mg) was refluxed with MeI (10 mL) and $\mathrm{Ag}_{2} \mathrm{O}(20 \mathrm{~mL})$ in DMF $(25 \mathrm{mg})$ for two days and then filtered. The filtrate was hydrolyzed with $10 \%$ ethanolic $\mathrm{H}_{2} \mathrm{SO}_{4}$ for $6-7 \mathrm{~h}$, to give methylated aglycone, identified as 5,7-dihydroxy- 3, 8, 4'-trimethoxy flavone and methylated sugars, which were identified as $2,3,4,6$-tetra- $O$-methyl- $D$-galactose $\left(\mathrm{R}_{\mathrm{G}} 0.89\right), 2$, 3, 4- tri- $O$-methyl- $L$-rhamnose $\left(\mathrm{R}_{\mathrm{G}} 1.02\right)$ and 2, 4-di- $O$-methyl- $L$-arabinose $\left(\mathrm{R}_{\mathrm{G}} 0.63\right)$.

\section{Enzymatic hydrolysis of compound 2}

Compound $2(15 \mathrm{mg})$ was dissolved in $\mathrm{MeOH}(20 \mathrm{~mL})$ and hydrolysed with equal volume of takadiastase enzyme. The reaction mixture was allowed to stay at room temperature for 2 days and filtered. The proaglycone and hydrolysate were studied separately. The hydrolysate was concentrated and subjected to paper chromatography examination using nBAW (4:1:5) as solvent, which showed the presence of $L$-rhamnose $\left(\mathrm{R}_{f} 0.36\right)$ and $L$-arabinose $\left(\mathrm{R}_{f}\right.$ 0.22) (Co-PC). The proaglycone was dissolved in $\mathrm{MeOH}(25 \mathrm{~mL})$ and further hydrolysed with equal volume of almond emulsin enzyme at room stemperature as usual procedure yielded aglycone, identified as 3, 5, 7-trihydroxy-8, 4'-dimethoxy flavone and sugar was identified as $D$-galactose $\left(\mathrm{R}_{f} 0.15\right)(\mathrm{Co}-\mathrm{PC})$.

\section{Antimicrobial activity of compounds 1 and 2}

The antibacterial activity of compounds $\mathbf{1}$ and $\mathbf{2}$ was determined by filter paper disc diffusion method ${ }^{9}$. The various gram (+ve) and gram (-ve) bacterial species were first incubated at $48^{\circ} \mathrm{C}$ for $42 \mathrm{~h}$. The sterile filter paper discs $(6 \mathrm{~mm})$ were soaked with standard antibacterial agent and various test samples and were dried at $50{ }^{\circ} \mathrm{C}$. The discs were then 
placed on soft nutrient agar (2\%) petri plates previously seeded with suspension of each bacterial species. The diameters of zone of inhibition were measured at $35 \pm 1{ }^{\circ} \mathrm{C}$ after $24 \mathrm{~h}$. The results are recorded in Table 1.

The antifungal activity of compounds was measured by PDA (Potato Dextrose Agar) with $4 \%$ agar for the preparation of plates and incubated with spores and mycelium suspension of fungi obtained from one week old culture. The diameters of zone of inhibition were measured at $26 \pm 1{ }^{\circ} \mathrm{C}$ after $46 \mathrm{~h}$. The results are recorded in Table 2.

Table 1. Antibacterial activity of compound 1 and $\mathbf{2}$

\begin{tabular}{ccccccccccc}
\hline & & \multicolumn{5}{c}{ Diameters of zone of inhibition mm } \\
\cline { 3 - 8 } S. & \multirow{2}{*}{ No } & Bacterial species & \multicolumn{3}{c}{$\begin{array}{c}\text { Concentration of } \\
\text { compound 1 \% }\end{array}$} & \multicolumn{3}{c}{$\begin{array}{c}\text { Concentration of } \\
\text { compound 2\% }\end{array}$} & \multirow{2}{*}{ Std, $^{* *}$} \\
\cline { 3 - 9 } & & 100 & 80 & 60 & 40 & 100 & 80 & 60 & 40 \\
\hline 1. & Escherichia coli & - & - & - & - & 6.54 & 3.56 & - & - & 11.25 \\
2. & Staphylococcus aureus & - & - & - & - & - & - & - & - & 34.33 \\
3. & Bacilius subtilis & - & - & - & - & - & 9.51 & - & - & 30.25 \\
4. & Micrococcus luteus & - & - & - & 4.66 & - & - & 3.12 & - & 10.5 \\
\hline
\end{tabular}

${ }^{*}$ The zone of inhibition $(\mathrm{mm})$ taken as average of four determination direction. ${ }^{* *}$ Ampicillin $(10 \mathrm{mg} / \mathrm{mL})$ used as standard antibacterial agent

Table 2. Antifungal activity of compound 1 and 2

\begin{tabular}{|c|c|c|c|c|c|c|c|c|c|c|}
\hline \multirow{3}{*}{$\begin{array}{l}\text { S. } \\
\text { No }\end{array}$} & \multirow{3}{*}{ Fungal species } & \multicolumn{9}{|c|}{ Diameters of zone of inhibition $\mathrm{mm}^{*}$} \\
\hline & & \multicolumn{4}{|c|}{$\begin{array}{c}\text { Concentration of } \\
\text { compound } 1 \%\end{array}$} & \multicolumn{4}{|c|}{$\begin{array}{l}\text { Concentration of } \\
\text { compound } 2 \%\end{array}$} & \multirow[t]{2}{*}{$\operatorname{Std}^{* * *}$} \\
\hline & & 100 & 80 & 60 & 40 & 100 & 80 & 60 & 40 & \\
\hline 1. & Candida albicans & - & - & 4.88 & - & - & - & - & 3.84 & 13.00 \\
\hline 2. & Rhizopus oryzae & - & - & - & - & - & - & - & - & 32.25 \\
\hline 3. & Aspergillus niger & - & - & - & - & 5.85 & - & - & - & 13.75 \\
\hline 4. & Mucor indicus & - & - & - & - & - & - & 4.24 & - & 10.00 \\
\hline
\end{tabular}

${ }^{*}$ The zone of inhibition (mm) taken as average of four determination direction. ${ }^{* * *}$ Ketocozole $(100 \mathrm{mg} / \mathrm{mL})$ used as standard antifungal agent. Study of compound 3

It was crystallized from acetone to give $600 \mathrm{mg}$. It has m.f. $\mathrm{C}_{21} \mathrm{H}_{20} \mathrm{O}_{11}$, m.p. $192-194{ }^{\circ} \mathrm{C}$, $[\mathrm{M}]^{+} 448$ (EIMS); found(\%), C 56.10, $\mathrm{H} 4.68$, calcd(\%) for m.f. $\mathrm{C}_{21} \mathrm{H}_{20} \mathrm{O}_{11}, \mathrm{C}$ 56.25, H 4.46; UV: $\lambda_{\max }(\mathrm{nm}):(\mathrm{MeOH}) 215,258,352$; IR: $v_{\max }^{\mathrm{KBr}}\left(\mathrm{cm}^{-1}\right), 3404,1662,1516 .{ }^{1} \mathrm{H}$ NMR $(90 \mathrm{MHz}$, DMSO- $\left.d_{6}\right), \delta(\mathrm{ppm}): 6.18(1 \mathrm{H}, \mathrm{d}, J=2.0 \mathrm{~Hz}, \mathrm{H}-6), 6.40(1 \mathrm{H}, \mathrm{d}, J=2.0 \mathrm{~Hz}, \mathrm{H}-8), 7.65(1 \mathrm{H}, \mathrm{d}, J$ $\left.=2.2 \mathrm{~Hz}, \mathrm{H}-2^{\prime}\right), 6.83\left(1 \mathrm{H}, \mathrm{d}, J=8.1 \mathrm{~Hz}, \mathrm{H}-5^{\prime}\right), 7.52\left(1 \mathrm{H}, \mathrm{dd}, J=8.4,2.0 \mathrm{~Hz}, \mathrm{H}-6^{\prime}\right), 4.10(1 \mathrm{H}, \mathrm{d}, J$ $\left.=1.3 \mathrm{~Hz}, \mathrm{H}-1^{\prime \prime}\right), 3.53\left(1 \mathrm{H}, \mathrm{m}, \mathrm{H}-2^{\prime \prime}\right), 3.64\left(1 \mathrm{H}, \mathrm{m}, \mathrm{H}-3^{\prime \prime}\right), 3.33\left(1 \mathrm{H}, \mathrm{m}, \mathrm{H}-4^{\prime \prime}\right), 4.26(1 \mathrm{H}, \mathrm{m}, \mathrm{H}-$ $\left.5^{\prime \prime}\right), 1.20\left(1 \mathrm{H}, \mathrm{d}, J=1.3 \mathrm{~Hz}, \mathrm{H}-6^{\prime \prime}\right) .{ }^{13} \mathrm{C}$ NMR (90 MHz, DMSO- $\left.d_{6}\right), \delta(\mathrm{ppm}): 156.60(\mathrm{C}-2)$, 134.36 (C-3), 177.84 (C-4), 161.40 (C-5), 98.90 (C-6), 164.36 (C-7), 93.94 (C-8), 157.35 (C-9), 104.28 (C-10), 121.26(C-1'), 115.62 (C-2'), 145.30 (C-3'), 148.52 (C-4'), 115.86 (C-5'), 121.28(C-6'), 101.92 (C-1"), 70.56 (C-2"), 70.72 (C-3"), 71.33 (C-4"), 70.15 (C-5"), 17.64 (C-6").

\section{Study of compound 4}

It was crystalysed from acetone to give $450 \mathrm{mg}$. It has m.f. $\mathrm{C}_{16} \mathrm{H}_{12} \mathrm{O}_{5}$, m.p. $260-262{ }^{\circ} \mathrm{C},[\mathrm{M}]^{+}$ 284 (EIMS); found(\%), C 67.20, H 3.85, calcd(\%) for m.f. $\mathrm{C}_{16} \mathrm{H}_{12} \mathrm{O}_{5}, \mathrm{C} 67.61, \mathrm{H} \mathrm{4.23,} \mathrm{UV:}$ $\lambda_{\max } \mathrm{nm}:(\mathrm{MeOH}) 265,308,324 ; \mathrm{IR}: v_{\max }^{\mathrm{KBr}}\left(\mathrm{cm}^{-1}\right), 3610,2980,2248,2128,1652,1598,1436$; 
${ }^{1} \mathrm{H}$ NMR (90 MHz, DMSO- $\left.d_{6}\right), \delta(\mathrm{ppm}): 6.76(1 \mathrm{H}, \mathrm{s}, \mathrm{H}-3), 6.88(1 \mathrm{H}, \mathrm{d}, J=2.4 \mathrm{~Hz}, \mathrm{H}-6), 6.48$ $(1 \mathrm{H}, \mathrm{d}, J=2.4 \mathrm{~Hz}, \mathrm{H}-8), 12.86(1 \mathrm{H}, \mathrm{s}, 5-\mathrm{OH}), 8.07\left(2 \mathrm{H}, \mathrm{d}, J=8.8 \mathrm{~Hz}, \mathrm{H}-2^{\prime}, 6^{\prime}\right), 7.14(2 \mathrm{H}, \mathrm{d}, J=$ $\left.8.8 \mathrm{~Hz}, \mathrm{H}-3^{\prime}, 5^{\prime}\right), 3.93\left(3 \mathrm{H}, \mathrm{s}, \mathrm{OCH}_{3}-4^{\prime}\right) .{ }^{13} \mathrm{C}$ NMR (90 MHz, DMSO- $\left.d_{6}\right), \delta(\mathrm{ppm}): 162.4(\mathrm{C}-2)$, 103.4 (C-3), 181.9 (C-4), 162.7(C-5), 99.95 (C-6), 163.5 (C-7), 94.3 (C-8), 156.5 (C-9), 105.6 (C-10), $122.2\left(\mathrm{C}-1^{\prime}\right), 128.9\left(\mathrm{C}-2^{\prime}, 6^{\prime}\right), 114.2\left(\mathrm{C}-3^{\prime}, 5^{\prime}\right), 161.4\left(\mathrm{C}-4^{\prime}\right), 55.8\left(\mathrm{OCH}_{3}-4^{\prime}\right)$.

\section{Study of compound 5}

It was crystallized from acetone to give $350 \mathrm{mg}$. It has m.f. $\mathrm{C}_{16} \mathrm{H}_{12} \mathrm{O}_{7}$, m.p. $186-188{ }^{0} \mathrm{C},[\mathrm{M}]^{+}$ 316 (EIMS); found (\%), C 60.36, H 3.64, calcd (\%) for m.f. $\mathrm{C}_{16} \mathrm{H}_{12} \mathrm{O}_{7}, \mathrm{C} 60.76, \mathrm{H} \mathrm{3.80}$; UV: $\lambda_{\max }(\mathrm{nm}):(\mathrm{MeOH}) 262,366 ;\left(+\mathrm{AlCl}_{3}\right)$ 266, 436; $\left(+\mathrm{AlCl}_{3} / \mathrm{HCl}\right) 264,420 ;(+\mathrm{NaOAc})$ 288, 372; IR: $v_{\max }^{\mathrm{KBr}}\left(\mathrm{cm}^{-1}\right), 3402,2976,1688,1638 .{ }^{1} \mathrm{H}$ NMR $\left(90 \mathrm{MHz}, \mathrm{DMSO}-d_{6}\right)$ : $\delta(\mathrm{ppm}) 6.22(1 \mathrm{H}, \mathrm{d}, J=2.1 \mathrm{~Hz}, \mathrm{H}-6), 6.46(1 \mathrm{H}, \mathrm{d}, J=2.1 \mathrm{~Hz}, \mathrm{H}-8), 9.43(1 \mathrm{H}, \mathrm{s}, 3-\mathrm{OH})$, $12.48(1 \mathrm{H}, \mathrm{s}, 5-\mathrm{OH}), 10.86(1 \mathrm{H}, \mathrm{s}, 7-\mathrm{OH}), 7.74\left(1 \mathrm{H}, \mathrm{d}, J=1.6 \mathrm{~Hz}, \mathrm{H}-2^{\prime}\right), 6.91(1 \mathrm{H}, \mathrm{d}, J=8.4$ $\left.\mathrm{Hz}, \mathrm{H}-5^{\prime}\right), 7.66\left(1 \mathrm{H}, \mathrm{dd}, J=8.6,1.4 \mathrm{~Hz}, \mathrm{H}-6^{\prime}\right), 9.75\left(1 \mathrm{H}, \mathrm{s}, 4^{\prime}-\mathrm{OH}\right), 3.19\left(3 \mathrm{H}, \mathrm{s}, 3^{\prime}-\mathrm{OCH}_{3}\right)$. ${ }^{13} \mathrm{C}$ NMR $\left(90 \mathrm{MHz}, \mathrm{DMSO}-d_{6}\right), \delta(\mathrm{ppm}): 146.5(\mathrm{C}-2), 136.3(\mathrm{C}-3), 176.5(\mathrm{C}-4), 161.4(\mathrm{C}-5)$, 98.7(C-6), 164.6 (C-7), 93.6 (C-8), 156.8 (C-9), 103.7 (C-10), 122.6 (C-1'), 111.7 (C-2'), $149.5\left(\mathrm{C}-3^{\prime}\right), 147.9\left(\mathrm{C}-4^{\prime}\right), 115.4\left(\mathrm{C}-5^{\prime}\right), 122.3\left(\mathrm{C}-6^{\prime}\right), 56.2\left(\mathrm{OCH}_{3}\right)$.

\section{Results and Discussion}

Chemical examination of methanolic extract of stems of Crotalaria medicaginea Lam., gave two new allelochemicals 1 and 2. Compound $\mathbf{1}$ (Figure 1) has molecular formula $\mathrm{C}_{33} \mathrm{H}_{40} \mathrm{O}_{21}$, m.p. 224-225 ${ }^{\circ} \mathrm{C},[\mathrm{M}]^{+} 772$ (FABMS). It gave Molisch and Shinoda tests ${ }^{10}$ showing its flavonoidal glycosidic nature. Its IR spectra showed strong absorption bands at 3400, 2924, $2848,1730,1465,1375,1269,1025,746,584,537$ and $428 \mathrm{~cm}^{-1}$. In UV spectrum two bands at $260 \mathrm{~nm}$ and $358 \mathrm{~nm}$ showed its flavonoidal skeleton. In ${ }^{1} \mathrm{H}$ NMR spectrum of compound 1 three singlets at $\delta 9.28, \delta 11.40$ and $\delta 10.74$ suggested the presence of $-\mathrm{OH}$ groups at C-3, C-5 and C-7 positions respectively. A singlet at $\delta 3.81$ confirmed the presence of -OMe group at C-6 position. Two singlets at $\delta 8.88$ and $\delta 8.94$ confirmed the presence of $-\mathrm{OH}$ groups at C-3' and C-4' positions. In ${ }^{1} \mathrm{H}$ NMR spectrum of the aglycone 1-A, a singlet at $\delta 6.48$ was assigned to $\mathrm{H}-8$ of A ring. A doublet at $\delta 6.84(1 \mathrm{H}, \mathrm{d}, J=8.1 \mathrm{~Hz})$ was assigned to $\mathrm{H}-5$ ' in ring $\mathbf{B}$. A doublet at $\delta 7.72(1 \mathrm{H}, \mathrm{d}, J=2.1 \mathrm{~Hz})$ and a double doublet $\delta 7.61(1 \mathrm{H}, \mathrm{dd}, J=8.4,2.1 \mathrm{~Hz})$ were assigned to $\mathrm{H}^{-2}$ ' and $\mathrm{H}^{-6} \mathrm{6}^{\prime}$ in ring $\mathbf{B}$ respectively. The anomeric proton signals at $\delta 5.32(1 \mathrm{H}$, br, s), $\delta 4.72(1 \mathrm{H}, \mathrm{d}, J=7.1 \mathrm{~Hz})$ and $\delta 4.62(1 \mathrm{H}, \mathrm{d}, J=7.6 \mathrm{~Hz})$ were assigned for H-1", H-1"' and $\mathrm{H}-1$ "'" of $L$-rhamnose, D-xylose and D-glucose respectively.

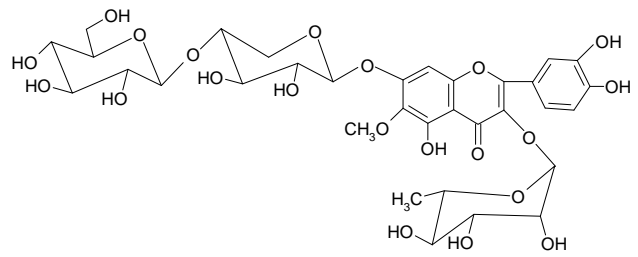

Figure 1. Compound 1

In the mass spectrum of the compound 1 , characteristic ion peaks at $m / z 772[\mathrm{M}]^{+}, 626$ $\left[\mathrm{M}^{+}\right.$-L-rhamnose $], 464\left[\mathrm{M}^{+}\right.$-D-glucose $]$and $332\left[\mathrm{M}^{+}-D\right.$-xylose, aglycone $]$ were found by subsequent losses from the molecular ion of each molecule of $L$-rhamnose, $D$-glucose and $D$-xylose showing $D$-glucose as terminal sugar, $D$-xylose was linked at C-7 position and $L$-rhamnose was attached at C-3 position of the aglycone. 
Acid hydrolysis of compound 1 with $10 \%$ ethanolic $\mathrm{H}_{2} \mathrm{SO}_{4}$ gave aglycone 1-A (Figure 2), m.p. 242-245 ${ }^{\circ} \mathrm{C}$, m.f. $\mathrm{C}_{16} \mathrm{H}_{12} \mathrm{O}_{8},[\mathrm{M}]^{+} 332$ (EIMS) and sugar moiety (ies). These were separated and studied separately. The aglycone 1-A was identified as 3, 5, 7, 3', 4'pentahydroxy-6-methoxy flavone by comparison of its spectral data with reported literature values $^{11}$.<smiles>COc1c(O)cc2oc(-c3ccc(O)c(O)c3)c(O)c(=O)c2c1O</smiles>

Figure 2. Compound 1-A

The aqueous hydrolysate after the removal of aglycone was neutralized with $\mathrm{BaCO}_{3}$ and $\mathrm{BaSO}_{4}$ filtered off. The filtrate was concentrated and subjected to paper chromatography examination and sugars were identified as $L$-rhamnose $\left(\mathrm{R}_{f} 0.36\right), D$-glucose $\left(\mathrm{R}_{f} 0.19\right)$ and $D$-xylose $\left(\mathrm{R}_{f} 0.27\right)(\mathrm{Co}-\mathrm{PC})^{12}$. Periodate oxidation of compound $\mathbf{1}$, confirmed that all the sugars were present in the pyranose form ${ }^{13}$.

The positions of sugar moieties in compound 1 were determined by permethylation ${ }^{14}$ followed by acid hydrolysis yielded methylated aglycone identified as 3, 7-dihydroxy-5, 6, 3', 4'-tetramethoxy flavone showed that glycosydation was involved at C-3 and C-7 positions of the flavanone and methylated sugars were identified as 2, 3, 4 -tri- $O$-methyl- $L$ rhamnose $\left(\mathrm{R}_{\mathrm{G}} 1.02\right), 2,3,4$, 6-tetra- $O$-methyl- $D$-glucose $\left(\mathrm{R}_{\mathrm{G}} 1.00\right)$ and 2, 3-di- $O$-methyl- $D$ xylose $\left(\mathrm{R}_{\mathrm{G}} 0.73\right)$ indicating that $\mathrm{C}-1$ "-OH of $L$-rhamnose was linked at C-3 position of the aglycone, C-1"'--OH of $D$-glucose was linked to C-4"'-OH of $D$-xylose and C-1"'-OH of $D$-xylose was attached with $\mathrm{C}-7$ position of the aglycone. Therefore it was concluded that interlinkage $(1 \rightarrow 4)$ was found between $D$-glucose and $D$-xylose which was further confirmed by ${ }^{13} \mathrm{C}$ NMR spectra (c.f. Experimental section).

Enzymatic hydrolysis of compound $\mathbf{1}$ with takadiastase enzyme liberated $L$-rhamnose $\left(\mathrm{R}_{f} 0.36\right)$ and proaglycone identified as $3,5,7,3$ ', 4'- pentahydroxy-6-methoxyflavone-7-O$\beta$ - $D$-glucopyranosyl- $(1 \rightarrow 4)-O-\beta$ - $D$-xylopyranoside showed the presence of $\alpha$-linkage between $L$-rhamnose and proaglycone. The proaglycone on further hydrolysis with almond emulsion enzyme liberated $D$-glucose $\left(\mathrm{R}_{\mathrm{f}} 0.19\right)$ followed by $D$-xylose $\left(\mathrm{R}_{\mathrm{f}} 0.27\right)$ and aglycone suggesting the presence of $\beta$-linkage between $D$-glucose and $D$-xylose as well as $D$-xylose and aglycone.

On the basis of above evidences the structure of compound 1 was characterized as 3 , $5,7,3^{\prime}, 4^{\prime}$-pentahydroxy-6-methoxyflavone-3- $O-\alpha-L$-rhamnopyranosyl-7- $O-\beta$ - $D$-glucopyranosyl$(1 \rightarrow 4)-O-\beta$ - $D$-xylopyranoside .

Compound 2 (Figure 3) has molecular formula $\mathrm{C}_{34} \mathrm{H}_{42} \mathrm{O}_{20}$, m.p. $234-235{ }^{\circ} \mathrm{C},[\mathrm{M}]^{+} 770$ (FABMS). It gave Molisch and Shinoda tests ${ }^{10}$ showing its flavonoidal glycosidic nature. Its IR spectra showed strong absorption bands at 3484, 2906, 2873, 1656, 1623, 1591, 926 and $852 \mathrm{~cm}^{-1}$. In UV spectrum two bands at $264 \mathrm{~nm}$ and $364 \mathrm{~nm}$ showed its flavonoidal skeleton. The bathochromic shift of $23 \mathrm{~nm}$ with $\mathrm{AlCl}_{3}$ and $35 \mathrm{~nm}$ with $\mathrm{NaOAc}$ in band I relative to methanol showed the presence of $-\mathrm{OH}$ groups at $\mathrm{C}-5$ and $\mathrm{C}-7$ positions in the aglycone $2-\mathrm{A}^{15,16}$. In ${ }^{1} \mathrm{H}$ NMR spectrum a singlet at $\delta 9.44$ confirmed the presence of $-\mathrm{OH}$ group at $\mathrm{C}-3$ position. Two singlets at $\delta 3.90$ and $\delta 3.86$ confirmed the presence of -OMe groups at C-8 and C-4' positions. A doublet at $6.15(1 \mathrm{H}, \mathrm{d}, J=2.0 \mathrm{~Hz})$ was assigned to $\mathrm{H}-6$ of A ring. Two doublets at $\delta 8.15(2 \mathrm{H}, \mathrm{d}, J=8.8 \mathrm{~Hz})$ were assigned to $\mathrm{H}-2^{\prime}$ and H-6' and two doublets at $\delta$ 
$7.12(2 \mathrm{H}, \mathrm{d}, J=8.8 \mathrm{~Hz})$ were assigned to $\mathrm{H}-3^{\prime}$ and $\mathrm{H}-5^{\prime}$ in ring $\mathbf{B}$. The anomeric proton signals at $\delta 5.23(1 \mathrm{H}, \mathrm{d}, J=7.5), \delta 4.96(1 \mathrm{H}, \mathrm{d}, J=6.9 \mathrm{~Hz})$ and $\delta 4.54(1 \mathrm{H}, \mathrm{d}, J=1.21 \mathrm{~Hz})$ were assigned for H-1", H-1"' and H-1"'' of $D$-galactose, $L$-arabinose and $L$-rhamnose respectively.

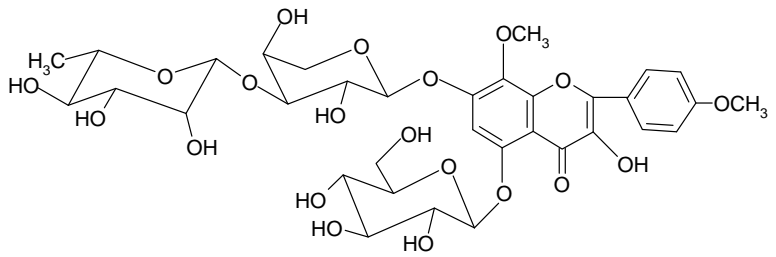

Figure 3. Compound 2

In the mass spectrum of the compound 2, characteristic ion peaks at $m / z 770[\mathrm{M}]+, 608$ [M $\mathrm{M}^{+}$-D-galactose], $462\left[\mathrm{M}^{+}-L\right.$-rhamnose $]$ and $330\left[\mathrm{M}^{+}-L\right.$-arabinose, aglycone $]$ were found by subsequent losses from the molecular ion of each molecule of $D$-galactose, $L$-rhamnose and $L$-arabinose revealing $L$-rhamnose as terminal sugar, $L$-arabinose was linked to aglycone at C-7 position and $D$-galactose was attached at C-5 position of aglycone.

Acid hydrolysis of compound 2 with $10 \%$ ethanolic $\mathrm{H}_{2} \mathrm{SO}_{4}$ gave aglycone 2-A (Figure 4), m.p. $256-257^{\circ} \mathrm{C}$, m.f. $\mathrm{C}_{17} \mathrm{H}_{14} \mathrm{O}_{7},[\mathrm{M}]^{+} 330$ (EIMS) and sugar moiety(ies). These were separated and studied separately. The aglycone 2-A was identified as 3, 5, 7-trihydroxy-8, 4'-dimethoxy flavones (c.f. Experimental section).<smiles>COc1ccc(-c2oc3c(OC)c(O)cc(O)c3c(=O)c2O)cc1</smiles>

Figure 4. Compound 2-A

The aqueous hydrolysate after the removal of aglycone was neutralized with $\mathrm{BaCO}_{3}$ and $\mathrm{BaSO}_{4}$ filtered off. The filtrate was concentrated and subjected to paper chromatography examination and sugars were identified as $D$-galactose $\left(\mathrm{R}_{f} 0.15\right), L$-rhamnose $\left(\mathrm{R}_{f} 0.38\right)$ and $L$-arabinose $\left(\mathrm{R}_{f} 0.23\right)(\mathrm{Co}-\mathrm{PC})^{12}$. Periodate oxidation of compound 2 , confirmed that all the sugars were present in the pyranose form ${ }^{13}$.

The positions of sugar moieties in compound 2 were determined by permethylation ${ }^{16}$ followed by acid hydrolysis yielded methylated aglycone identified as 5, 7-dihydroxy-3, 8, 4'-trimethoxy flavone showed that glycosydation was involved at C-5 and C-7 positions of the flavone and methylated sugars were identified as 2, 3, 4, 6 -tetra- $O$-methyl- $D$-galactose $\left(\mathrm{R}_{\mathrm{G}} 0.89\right), 2,3$, 4-tri- $O$-methyl- $L$-rhamnose $\left(\mathrm{R}_{\mathrm{G}} 1.02\right)$ and 2 , 4-di- $O$-methyl- $L$-arabinose $\left(\mathrm{R}_{\mathrm{G}}\right.$ 0.65 ) indicating that $\mathrm{C}-1$ "-OH of $D$-galactose was linked to $\mathrm{C}-5$ position of the aglycone, $\mathrm{C}-1$ "'"-OH of $L$-rhamnose was linked to C-3"'-OH of $L$-arabinose and $\mathrm{C}-1$ "'-OH of $L$-arabinose was attached with $\mathrm{C}-7$ position of the aglycone. Therefore, it was concluded that interlinkage $(1 \rightarrow 3)$ between $L$-rhamnose and $L$-arabinose was found which was further confirmed by ${ }^{13} \mathrm{C}-\mathrm{NMR}$ spectra (c.f. Experimental section).

Enzymatic hydrolysis of compound 2 with takadiastase enzyme liberated $L$-rhamnose $\left(\mathrm{R}_{f} 0.38\right)$ followed by $L$-arabinose $\left(\mathrm{R}_{f} 0.22\right)$ and proaglycone identified as 3, 5, 7-trihydroxy8 , 4'-dimethoxyflavone-5- $O-\beta$ - $D$-galactopyranoside showed the presence of $\alpha$-linkage between $L$-rhamnose and $L$-arabinose as well as between $L$-arabinose and proaglycone. Proaglycone on further hydrolysis with almond emulsin enzyme liberated $D$-galactose $\left(\mathrm{R}_{\mathrm{f}} 0.15\right)$ and aglycone suggesting the presence of $\beta$-linkage between $D$-galactose and aglycone. 
On the basis of above evidences the structure of compound 2 was characterized as 3, 5, 7-trihydroxy-8,4'-dimethoxyflavone-5- $O-\beta$ - $D$-galactopyranosyl-7- $O-\alpha-L$-rhamnopyranosyl$(1 \rightarrow 4)-O-\alpha$ - $L$-arabinopyranoside.

Compound 3 (Figure 5) has m.p.192-194 ${ }^{\circ} \mathrm{C}$, m.f. $\mathrm{C}_{21} \mathrm{H}_{20} \mathrm{O}_{11},[\mathrm{M}]^{+} 448$ (EIMS). It was characterized as quercitrin by comparison of its spectral data with reported literature values $^{17-19}$.

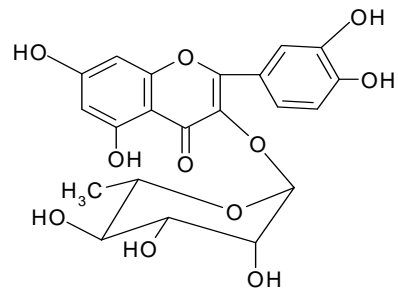

Figure 5. Compound 3

Compound 4 (Figure 6) has m.p. $260-262{ }^{\circ} \mathrm{C}$, m.f. $\mathrm{C}_{16} \mathrm{H}_{12} \mathrm{O}_{5}$, [M] ${ }^{+} 284$ (EIMS). It was identified as acacetin by comparison of its spectral data with reported literature values ${ }^{20-21}$.<smiles>COc1ccc(-c2cc(=O)c3c(O)cc(O)cc3o2)cc1</smiles>

Figure 6. Compound 4

Compound 5 (Figure 7) has m.p.186- $187{ }^{\circ} \mathrm{C}$, m.f. $\mathrm{C}_{16} \mathrm{H}_{12} \mathrm{O}_{7},[\mathrm{M}]^{+} 316$ (EIMS). It was identified as isorhamnetin by comparision of its spectral data with reported literature values $^{22,23}$.<smiles>COc1cc(-c2oc3cc(O)cc(O)c3c(=O)c2O)ccc1O</smiles>

Figure 7. Compound 5

Compounds $\mathbf{1}$ and $\mathbf{2}$ were screened for antibacterial and antifungal activity against various gram (+ve) gram (-ve) bacteria and fungi. The results reported in Table 1 showed that compound $\mathbf{1}$ was found to be active against gram bacteria Micrococcus luteus (+ve) at lower concentration and no activity was found against E.coli(-ve), Staphyloccus aureus (+ve) and Bacilius subtilis (+ve). Compound $\mathbf{2}$ has also shown less activity against bacteria E. coli (-ve), Bacilius subtilis $(+\mathrm{ve})$ and Micrococcus luteus $(+\mathrm{ve})$. In case of antifungal activity compound 1 showed activity against Candida albicans and no activity was found against Rhizopus oryzae, Aspergillus niger and Mucor indicus. Compound $\mathbf{2}$ showed less activity against Candida albicans, Aspergillus niger and Mucor indicus at lower concentration.

\section{Conclusion}

The phytochemical analysis of methanolic extract of Crotalaria medicaginea Lam. shown the presence of two new allelochemicals. These compounds showed antibacterial and antifungal activity against various gram positive and gram negative bacteria and fungi. The results also suggested the medicinal importance of the plant. The plant could serve as useful sources for new antimicrobial agents. 


\section{Acknowledgement}

Authors are thankful to the Director, CDRI Lucknow (U.P.) for recording various spectral data, Director General, M.P.C.S.T., Bhopal (M.P.) for providing facilities for antimicrobial activity and Head, Department of Chemistry, Dr. H.S. Gour Central University, Sagar (M.P.) for providing necessary laboratory facilities.

\section{References}

1. Kirtikar K R and Basu B D, Indian Medicinal Plants, $2^{\text {nd }}$ Edition Allahabad, 1975, 2, 696-697.

2. Asolkar LV, Kakkar K K and Chakre O J, Glossary of Indian Medicinal Plants with Active Principles, 2000, part I (A-K), 239.

3. The Wealth of India, Dictionary of Indian Raw Materials and Industrial Products, 1950, II, 382.

4. Rao G V R, Rao P S and Raju K R, Phytochemistry, 1987, 26(10), 2866-2868; DOI:10.1016/S0031-9422(00)83612-2

5. Gupta A K and Bemiller J N, Phytochemistry, 1990, 29(3), 853-855; DOI:10.1016/0031-9422(90)80032-C

6. Fletcher M T, Hayes P Y, Somerville M J and De Voss J J, J Agr Food Chem., 2011, 59(21), 11888-11892; DOI:10.1021/jf203147x

7. Kaur N, Singh J and Kamboj S S, Indian J Biochem Biophys., 2002, 39(1), 49-54.

8. Fletcher M T, Mckenzie R A, Blaney B J and Reichmann K G, J Agr Food Chem., 2009, 57(1), 311-319; DOI:10.1021/jf8026099

9. Casida L E, Industrial Microbiology, John Willey and Sons Ltd, New York, 1961.

10. Shinoda J, J Pharm Soc Jpn., 1928, 48, 214.

11. Guillermo Schmeda H, Alejandro T, Cristina T, Jaime R, Susana L and Gabrla E F, Z Naturforsch, 2004, 59c, 345-353.

12. Lederer E and Lederer M, Chromatography, Elsevier Publishing Company, New York, 1957, 1, 247.

13. Hakomoni S, J Biochem., 1965, 66, 205-207.

14. Petek F, Bull Soc Chem Fr., 1965, 68, 263-268.

15. Mabry T J, Markham K R and Thomas M B, The Systematic Identification of Flavonoids, Springer, New York, 1970, 51, 258.

16. Ulubelen A, Timmermann B N and Mabry T J, Phytochemistry, 1980, 19(5), 905-908; DOI:10.1016/0031-9422(80)85136-3

17. Park S, Yang S, Ahn D, Yang J H and Kim D K, J Korean Soc Appli Biol Chem., 2011, 54(5), 685-692; DOI:10.1007/BF03253146

18. Subhadhirasakul S, Jankeaw B and Malinee A, Songklanakarin J Sci Technol., 2003, 25(3), 351-357.

19. Koolen H H F, Soares E R, Da Silva F M A, De Souza A Q L, Filho E R and De Souza A D L, Brazilian Journal of Pharmacognosy, 2012, 22(1), 189-192.

20. Miyazawa M and Hisama M, Biosci Biotechnol Biochem., 2003, 67(10), 2091-2099.

21. Kim Y C and Kingston D G I, Korean J Pharmacogn., 1995, 26(3), 248-252.

22. Yang X W and Teng J, J Chin Pharm Sci., 2007, 16(1), 20-23.

23. Owolabi M A, Coker H A B and Jaja S I, J Med Plants Res., 2010, 4(12), 1130-1135; DOI:10.5897/JMPR09.429 